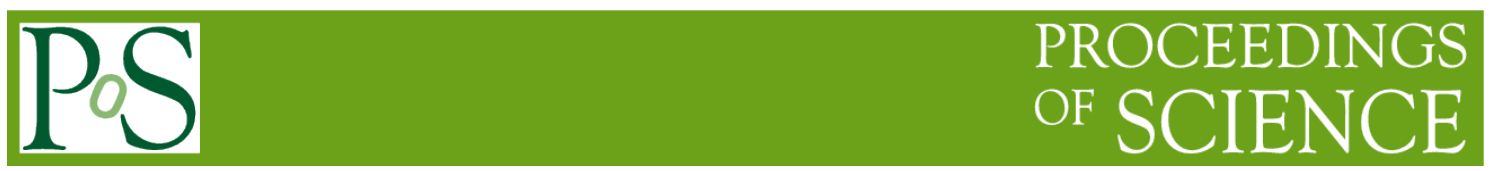

\title{
Neutron position sensitive detector
}

\author{
Kirin D.Yu. ${ }^{1}$, Chernishov O.A., Goryachev V.S., Mikhailov K.R., Polozov P.A., Prokudin \\ M.S., Romanov D.V., Sharkov G.B., Stavinskiy A.V., Stolin V.L., Zhigareva N.M. \\ Institute of theoretical and experimental physics (ITEP) \\ Moscow, Russia \\ E-mail: Dmitriy.Kirineitep.ru
}

Abstract.

Position-sensitive neutron detector, developed at ITEP is presented. The report includes results of beam tests for the first prototype. Based on beam tests the spatial resolution is defined and detector shape is optimized. The principal restriction for neutron position accuracy is extracted from the results of computer simulation in GEANT4 framework. Expected spatial resolution of the detector to be created is less than $1.5 \mathrm{~cm}$.

XXII International Baldin Seminar on High Energy Physics Problems

15-20 September 2014

JINR, Dubna, Russia

${ }^{1}$ Speaker 


\section{Introduction}

Physical motivation to create neutron detector:

1. Neutron is one of main particle specie for nucleus nuclear collisions at energy range ${\sqrt{\mathrm{S}_{\mathrm{NN}}}}<10 \mathrm{GeV}$ (Nuclotron, FAIR, NICA)

2. State of nuclear matter depends on neutron/proton ratio.

3. To identify some strange particle one needs to identify neutrons (for example $\Sigma^{+} \rightarrow \mathrm{n} \pi^{+}$).

4. In femtoscopy measurements space-time parameters for $\mathrm{n} / \mathrm{n}, \mathrm{p} / \mathrm{p}$ and $\mathrm{n} / \mathrm{p}$ pairs are different.

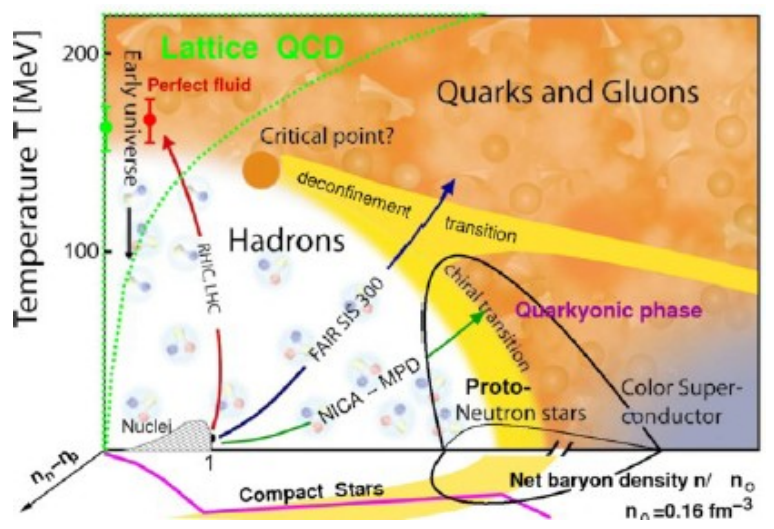

figure 1. Phase diagram

The design goal for identified neutron energy range is from 10 to $200 \mathrm{MeV}$. This kinetic energy range is motivated typical temperature of nuclear matter of the order of $100 \mathrm{MeV}$. Neutron momentum accuracy can be estimate, for example, from femtoscopy measurements. The width of femtoscopy effect of the order of $30 \mathrm{MeV} / \mathrm{c}$. So, the necessary momentum accuracy is about $10-15 \mathrm{MeV} / \mathrm{c}$.

There is so-called cross-talk problem in the femtoscopy measurements with neutrons.

\subsection{Cross-Talk problem}

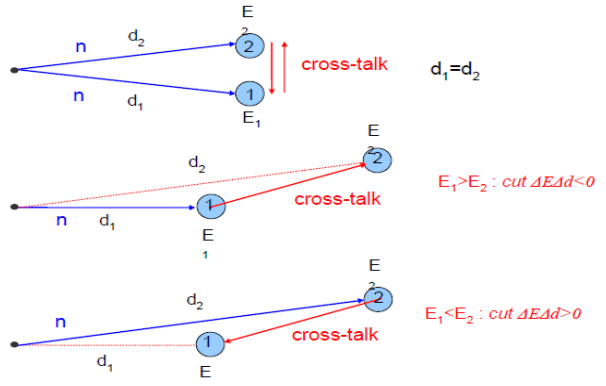

figure 2. changed distance between modules

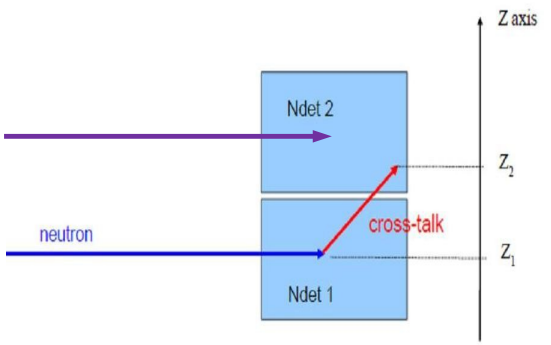

figure 3. Cross-talk effect

If the same neutron is registered in two or more modules. It's called cross-talk effect. It simulated registration of two or more neutrons in neighbor modules. This effect is important and dangerous for femtoscopy measurements. To avoid this effect one can put modules at different distance, but this method need a lot of space(see fig.2). Another solution - position sensitive detector(see fig. 3). 


\subsection{Requred features}

So, we can formulate required features for new detector: neutron kinetic energy range 10-200 MeV, measurement accuracy for neutron momentum $10-15 \mathrm{MeV} / \mathrm{c}$, the modular structure of the detector for correlation measurements, position resolution(about $1 \mathrm{~cm}$ ) much better than the module size (about 10-20 $\mathrm{cm}$ ), compact module.

\section{First prototype}

The first prototype of the neutron detector created ITEP is shown in the pictire (fig.4). It consist of a plastic scintillator and waveshift fiber in the corners that collect light to silicon avalanche photodiode (APD). Veto counter consist from $5 \mathrm{~mm}$ scintillator, fiber and diode. Veto counter is used for the detection of charged particles.
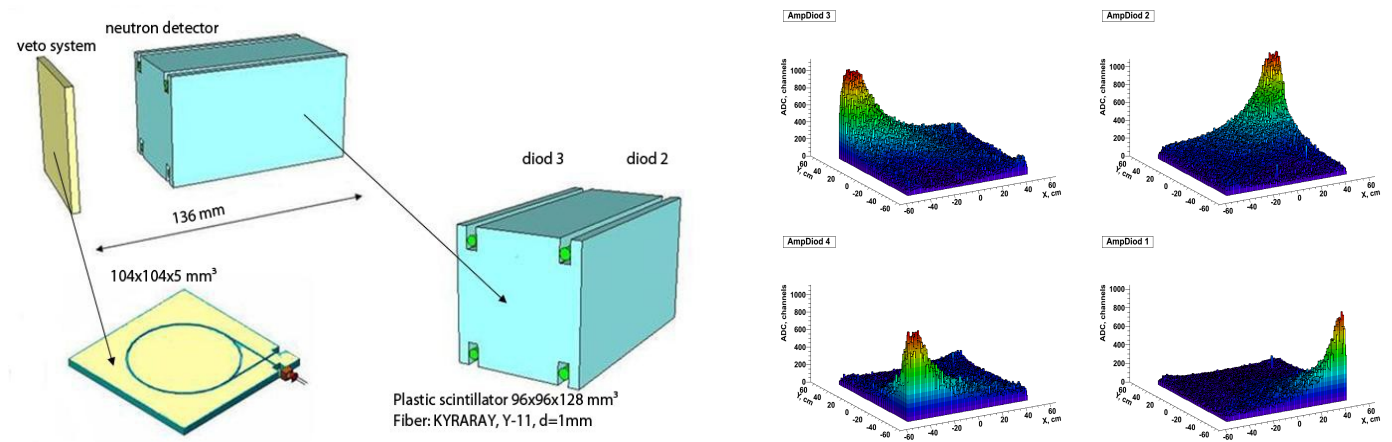

figure 4. First prototype

figure 5. Dependence of the signal amplitude on the protons coordinate

\subsection{Beam tests}

First beam tests were done with proton beam at $3 \mathrm{GeV} / \mathrm{c}$ energy at ITEP. Detector was placed between two proportional chambers, which defines the coordinates with an accuracy of about $1 \mathrm{~mm}$. For the main detector, one can see the dependence of the signal amplitude on the protons coordinate. The closer particle to diode, the stronger the signal(fig. 5).

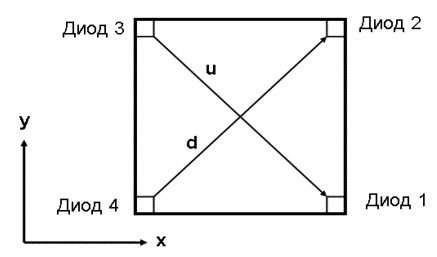

figure 6. Coordinates d, $u$

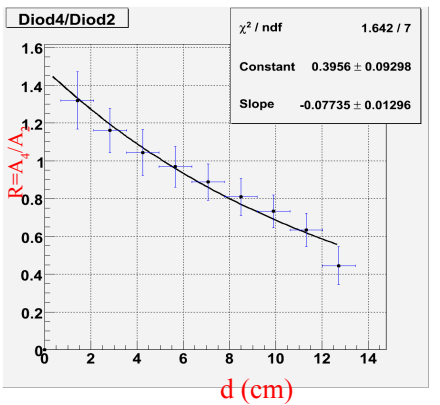

figure 7. Dependence of the A4/A2 from d

To estimate the spatial resolution we explored the the dependence of the amplitude ratio of the fourth and second diodes from coordinates d (fig.6, 7). We obtained the spatial resolution for this prototype about $2,5 \mathrm{~cm}$ (fig.8). 

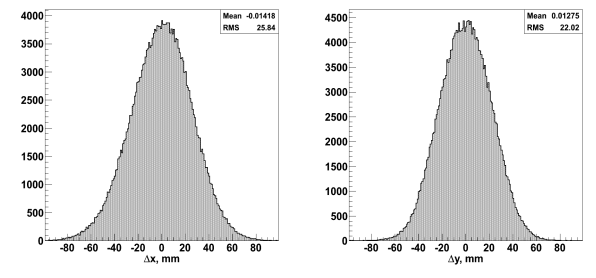

figure 8. Amplitude spectrum

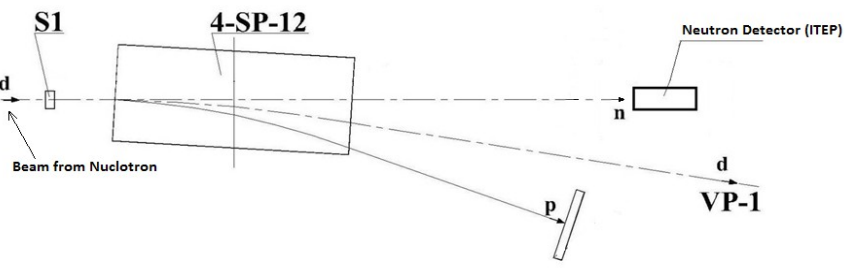

figure 9. Experimental setup

Prototype 1 been tested also on neutron beam at Nuclotron. On the fig.9 experimental setup is shown. We used deutron beam and carbon target for stripping. Proton was deviated in magnetic field. And neutron was identified by the detector.

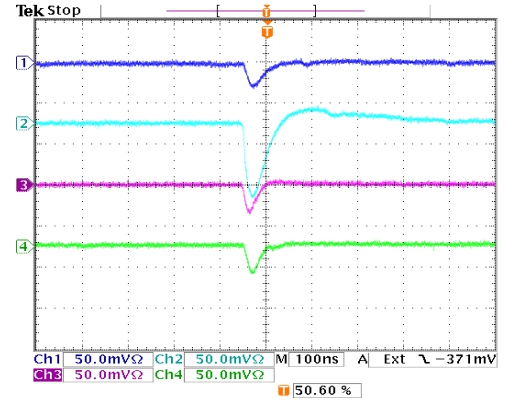

figure 10. Event where interaction point point is near diode 2

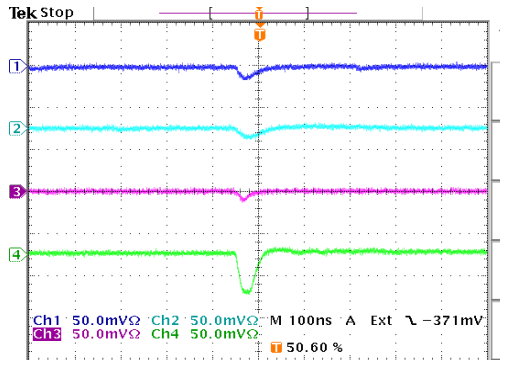

figure 11. Event where interaction is near diode 4

Two different events are shown:

the first figure corresponds to event where interaction point is near diode 2(fig.10); the second one corresponds to event where interaction point is near diode 4(fig.11). One can see that the largest amplitude corresponds to the closet to the beam diode.

\section{Second prototype}

To improve the spatial resolution the detector was modified. New shape was chosen for the detector - hexagon. 4 fibers located in each corner. The thickness of the new module is 20 $\mathrm{cm}$. As the result of this modification we expect the number photons will in 6 times large then for the first prototype. Some photos which was done during the assembling of the second prototype is shown on figure 12 .
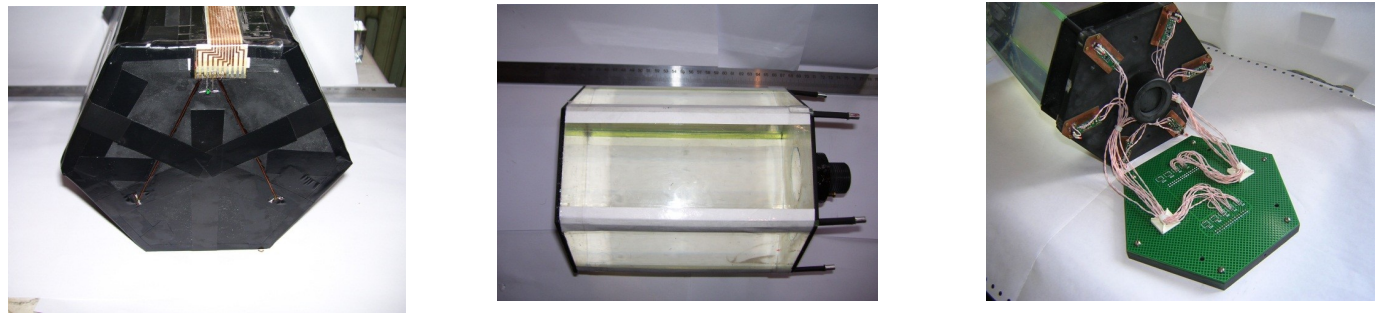

figure 12. Second prototype during the assembling 


\subsection{Simulation}

Simulation was made within GEANT4 framework to determine the principal limitation for neutron position accuracy. Neutron collisions with detector material was simulated. We used material with hydrogen to carbon ratio like BC400. Simulations was made for different neutron energies. Statistic for each neutron energies was one hundred thousand events. Events in which one proton is produced in the first interaction have been selected. In the fig. 13 we show side view for events with fixed initial energy of neutron $150 \mathrm{MeV}$ but different energy of recoil proton (red line - proton, green - neutron, blue - electron).

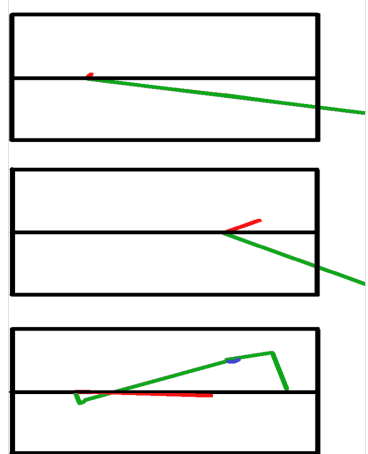

figure 13. Particle trajectories
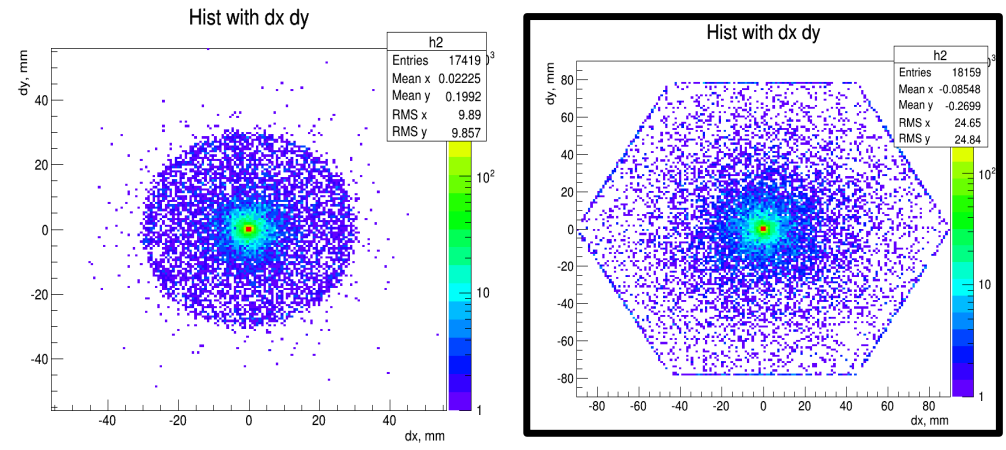

figure 14. Distributions of the stop point position for the secondary protons

The figure 14 shows the distributions of the stop point position for the secondary protons in the transverse coordinates. Initial neutron energy 150 (left side of picture) and 300 $\mathrm{MeV}$ (right side of picture). In the picture you can see that for $300 \mathrm{MeV}$ part of neutrons fly away over side walls of the detector. For neutron initial energy $150 \mathrm{MeV}$ most of simulate events concentrate within a circle, and you can see the concentration near the boundary of this circle, which will be explained later. Figure 15 shows the maximum deviation of the protons



figure 15. Maximum deviation of the protons from the trajectory of initial neutron from the trajectory of initial neutron. If neutron to proton transferred energy is small, proton range is small and it stops near the initial trajectory of neutron. If neutron to proton transferred energy is close to neutron initial energy proton trajectory is close to the trajectory of the neutron. The maximum deviation is achieved at an energy of $100-120 \mathrm{MeV}$, which corresponds to the boundary of circle at figure 14 .

\begin{tabular}{|l|c|c|c|c|c|}
\hline Neutron energy, MeV & 50 & 100 & 150 & 200 & 300 \\
\hline $\begin{array}{l}\text { mean deviation of } \\
\text { protons track, mm }\end{array}$ & 0,6 & 2,4 & 4,8 & 7,5 & 12,2 \\
\hline
\end{tabular}

\section{table 1. Results of simulation}

Mean deviation smaller then $1 \mathrm{~cm}$ for initial neutron energy less $200 \mathrm{MeV}$ (see table 1). 




Figure 16 shows simulated amplitude dependence on initial neutron coordinates. We obtained relative deviation from the mean amplitude $\sigma=0,056$.

\section{figure 16. Amplitude dependence on initial neutron coordinates}

As the next step we plan to build the detector with a photomultiplier and a veto couter. Such a modification allows to achieve a high time resolution (better than 200 ps) and the coordinate resolution remains as high as it was.

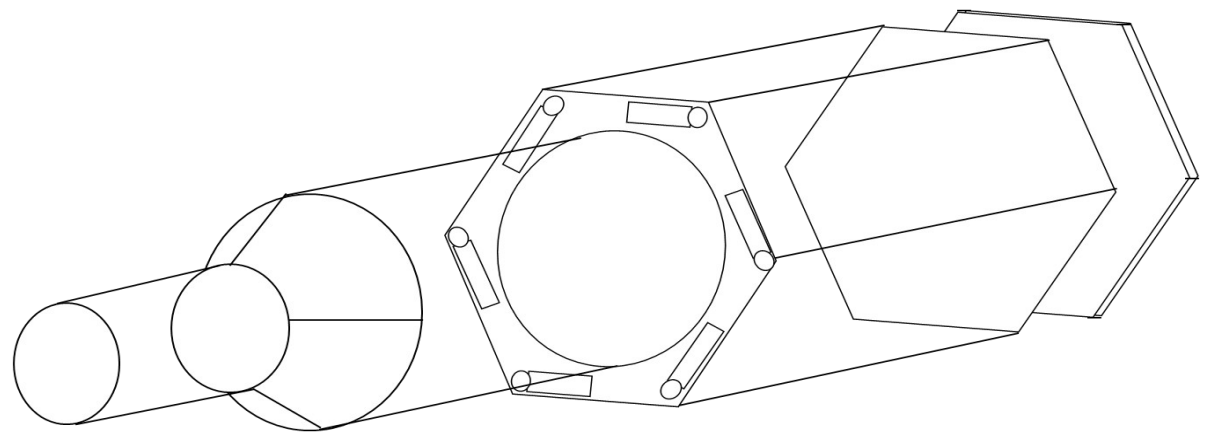

figure 17. Prototype 3 with photomultiplier and veto counter

\section{Conclusions}

1. The prototype 1 was designed, constructed and tested. Beam tests was made at ITEP(2011) and JINR(2012-2013).

2. The results of beam tests was used in simulations of the prototype 2 .

3. All characteristics of the prototype 2, obtained from this simulations, are in accordance with designed goals.

4. Prototype 2 is constructed and ready for the beam test at Nuclotron (MARUSYA).

This research has been carried out within the scope of the ERG (GDRE) "Heavy ions at ultrarelativistic energies", a European Research Group comprising IN2P3/CNRS, authors also acknowledge support by the RFBR-CNRS grant No 14-02-93108 and RFBR grant 14-02-00896.

\section{References}

[1]G. B. Dzubenko «Position-sensitive neutron detector», IV Cherenkov Readings: New Methods in Experimental Nuclear Physics and Particle Physics (Moscow, LPI, 12 april 2011 ) page 56.

[2]http://www.cpta-apd.ru/RUdoc/Low-noise\%20amplifier.html

[3] http://www.cpta-apd.ru/RUdocAPD/CPTA\%20MRS-APD\%20avalanche\%20photodiode $\% 20$ ru149-35.html

[4]http://geant4.web.cern.ch/geant4/

[5]http://prod.detectors.saint-gobain.com/uploadedFiles/SGdetectors/Documents

/Product_Data_Sheets/BC400-404-408-412-416-Data-Sheet.pdf 